\title{
Theme: Pediatric Hematology
}

\begin{abstract}
Diagnosis of iron deficiency anemia using densitybased fractionation of red blood cells (Lab Chip. 2016;16:3929-39).
\end{abstract}

A coulter is expensive, not portable, and requires trained personnel; it is unavailable in many low-resource settings. The paper describes a low-cost and rapid method to diagnose iron deficiency anemia (IDA) using aqueous multiphase systems (AMPS). AMPS are preloaded into a microhematocrit tube and used with a drop of blood from a fingerstick. After two minutes in a low-cost centrifuge, the tests were read by eye with a sensitivity of $84 \%$ and a specificity of $78 \%$. The AMPS test outperformed diagnosis by hemoglobin alone, and was comparable to reticulocyte hemoglobin concentration.

The authors stated that they have created a simple/low-cost method to detect microcytic and hypochromic red blood cells, and hence IDA. Instead of directly measuring hemoglobin or serum ferritin, this new method relied on observing the way in which red blood cells move through a viscous media (a function of their density, size and shape) to make a diagnosis.

Hematologic outcomes after total or partial splenectomy for congenital hemolytic anemia ( $J$ Pediatr Surg. 2016;51:122-7)

The risks associated with total splenectomy (TS) such as postsplenectomy sepsis and venous thromboembolism are major concerns. It has prompted consideration for partial splenectomy (PS), with the goal of removing enough spleen to gain a desired hematologic effect while preserving splenic immune function. The purpose of the multicentric study from USA/Canada was to define the hematologic response to TS or PS in children with hereditary spherocytosis (HS) or sickle cell disease (SCD). The analysis included 130 children, with 62\% $(n=81)$ undergoing TS. For children with HS, all hematologic measures improved after TS, including a $4.1 \mathrm{~g} / \mathrm{dL}$ increase in hemoglobin. Hematologic parameters also improved after PS, although the response was less robust (hemoglobin increase $2.4 \mathrm{~g} / \mathrm{dL}, P<0.001$ ). For children with $\mathrm{SCD}$, there was no change in hemoglobin. These data offer guidance to families and clinicians considering TS or PS.

A randomized trial of amlodipine in addition to
standard chelation therapy in patients with
thalassemia major (Blood. 2016;128:1555-61)

Cardiovascular disease resulting from iron accumulation is a major cause of death in patients with thalassemia major. Voltagegated calcium-channel blockade prevents iron entry into cardiomyocytes, and may provide an adjuvant treatment to chelation by reducing myocardial iron uptake. In a novel approach, the authors from Brazil evaluated whether addition of amlodipine to chelation strategies would reduce myocardial iron overload in thalassemia major. In a multicenter, double-blind, randomized, placebo-controlled trial, 62 patients were allocated to receive oral amlodipine or placebo in addition to their current chelation regimen. The main outcome was change in myocardial iron concentration (MIC) determined by T2* MRI at 12 months. Authors concluded that in patients of thalassemia with cardiac siderosis, amlodipine combined with chelation therapy reduced cardiac iron more effectively than chelation therapy alone.

Hematopoietic stem cell transplantation for
$\begin{aligned} & \text { homozygous â-thalassemia and â-thalassemia/ } \\ & \text { hemoglobin E patients from haploidentical donors } \\ & \text { (Bone Marrow Transplant. 2016;51:813-8) }\end{aligned}$

Full-house HLA related or unrelated matched donors are often unavailable for the curative treatment of hematopoietic stem-cell transplant (SCT) in patients with thalassemia major. The authors, from Thailand, explored the use of a mismatched-related ('haplo') donor in 31 patients. Eleven patients received SCT from the father and 20 patients from the mother. All patients received two courses of pre-transplant immunosuppressive therapy. A conditioning regimen was given followed by T-cell-replete peripheral blood progenitor cells. The median age was 10-years (range: 2-20). Twenty-nine patients engrafted with $100 \%$ donor chimerism. Two patients suffered primary graft failure. A reversible veno-occlusive disease, acute GvHD and limited-chronic GvHD was observed in 5,9 and 5 patients, respectively. Projected overall and event-free survival rates at 2 years were $95 \%$ and $94 \%$, respectively. The median follow-up time was 12 months (range, 7-33 months).

The study demonstrated that outcomes after Haplo-SCT in patients with thalassemia are acceptable. Finding need to be confirmed in a larger series of patients, to gain confidence of paediatricians in recommending 'haplo' transplant to patients with thalassemia major.

Iron-refractory Iron Deficiency Anemia (IRIDA): A heterogeneous disease that is not always iron refractory (Am J Hematol. 2016 Sep 19. [Epub ahead of print])

IRIDA is a relatively newly described, autosomal recessive disease. It is characterized by microcytic, hypochromic anemia and raised serum hepcidin levels. The patients typically present in childhood with microcytic anemia not responding to oral iron, along with remarkably low transferrin saturation. Serum ferritin levels are generally within the low normal range. Although the genetic basis of IRIDA was elucidated only recently, the disorder was first described in the early 1980s. Much is still unknown about its pathophysiology, genotype-phenotype correlation and optimal clinical management. It is likely that this condition is underdiagnosed. The disease is due to mutations in the TMPRSS6 gene encoding Matriptase 2, a trans-membrane serine protease that plays an essential role in down-regulating hepcidin. The authors have described 14 different TMPRSS6 variants in 21 phenotypically affected IRIDA patients from 20 families living in the Netherlands.

In the coming years, it is likely that more will be known about the prevalence and pathophysiology of this disorder.

DEEPAK BANSAL deepakbansaldr@gmail.com 